\title{
A EXPERIÊNCIA ARGENTINA DE OFERTA DE ENSINO SUPERIOR NO SISTEMA PRISIONAL - ENTREVISTA COM ANALÍA UMPIERREZ
}

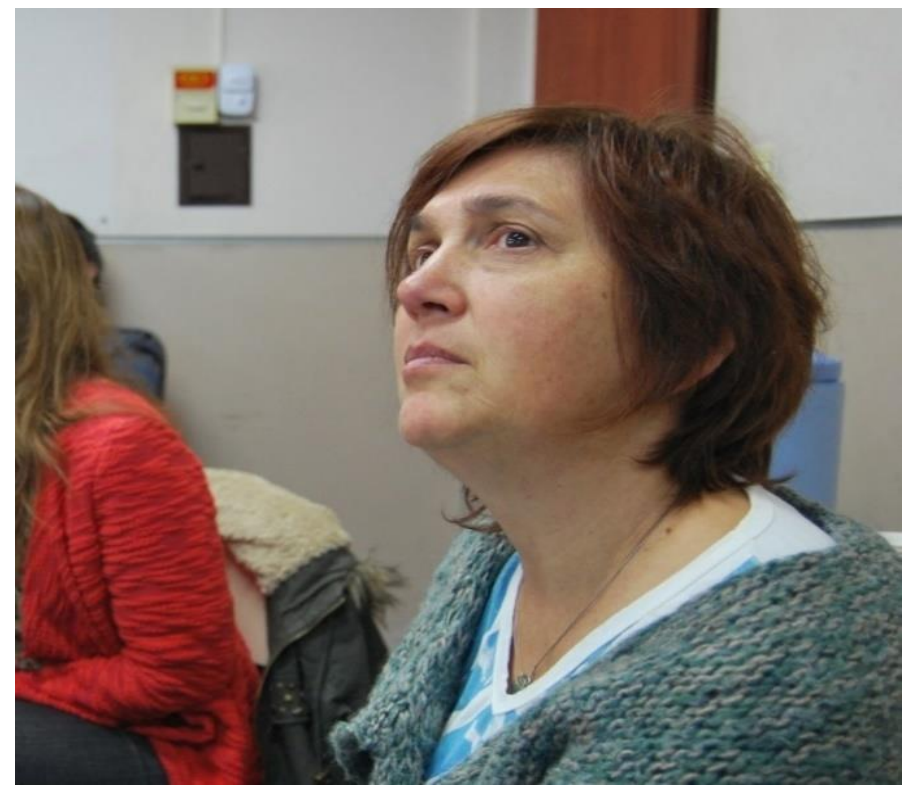

O direito à educação de pessoas privadas de liberdade é um desafio nos países da América do Sul. Experiências diversas buscam avançar no compromisso assumido em tratados internacionais, como a $\vee$ Confintea, por exemplo, de ampliar o acesso à escola básica, mas ainda os países estão distantes da efetiva garantia desse direito. Para se ter ideia do quão distantes estamos no Brasil, a escola básica atinge aproximadamente $10 \%$ da população prisional, embora a demanda potencial, conforme dados do Sistema Nacional de Informação Penitenciária - INFOPEN (2018) seja de quase $80 \%$. Nesse cenário, parece ainda mais distante das pessoas privadas de liberdade a chance de acessar o ensino superior.

Em oposição a uma realidade de negligência e violação de direitos nos espaços de privação de liberdade, comum entre Brasil e Argentina, este país desenvolve experiências bastante particulares, de oferta de ensino superior dentro do cárcere, por meio da realização de cursos de graduação e de programas de extensão universitária.

A presença da Universidad Nacional del Centro de la Província de Buenos Aires - UNICEN em estabelecimentos penais iniciou nos anos 1990, porém a criação do seu programa de extensão Universidad en la Cárcel foi impulsionada pela promulgação da Lei n. 26.695, embora já existissem atividades das universidades em unidades penais argentinas. Esta altera a Lei de Execução Penal da Argentina e inclui um artigo sobre a remição de pena pelo estudo que prevê o acesso ao ensino superior (UMPIERREZ, 2016). 
Analía Umpierrez é coordenadora deste projeto na Universidad Nacional del Centro de la Província de Buenos Aires. Doutora em educação, ela também coordena o Programa Educación en contextos de encierro da Facultad de Ciencias Sociales (UNICEN).

Nesta entrevista, a docente e pesquisadora resgata a história do ensino superior no cárcere em seu país e, assim, contextualiza o direito à educação e especificamente ao ensino superior pelas pessoas privadas de liberdade na Argentina. Reflete sobre os avanços, impasses e desafios dessa experiência no atual contexto político, de avanço do neoliberalismo e de grupos reacionários não só na Argentina, mas na América Latina de modo geral.

As reflexões desta entrevista contribuem para compreender a educação em contextos de privação de liberdade na perspectiva da garantia de direitos, na medida em que apresenta experiências exitosas e oferece ao leitor e leitora brasileires um retrato daquilo que ainda não conseguimos alcançar nas políticas educacionais brasileiras no que se refere ao direito à educação no sistema prisional do país.

Entrevistadores - Para empezar, ¿es posible hablar sobre su trayectoria académica y profesional?

Analía - Bueno, yo soy egresada de la carrera de ciencias de la educación, soy licenciada y profesora en ciencias de la educación. Trabajé muchos años como orientadora educacional en escuelas primarias y en la formación de dccentes. Trabajé al principio de mi carrera en la universidad, luego me fui y desde el 1997, volví a la Universidad, cuando se abrieron carreras docentes en la Facultad de Ciencias Sociales en la ciudad en la que vivo. Empecé una maestría que me tomó muchos años, porque en el medio tuve hijos y siempre me pareció más interesante dar clases al principio de mi carrera que investigar. Sin embargo, a lo largo de los años descubrí que me interesaba investigar y que no se puede pensar la enseñanza separada de la investigación. Luego, no hace tanto, empecé mi doctorado y finalmente lo terminé el año pasado. Trabajo como profesora en formación docente en la Universidad y también en una materia de la licenciatura en Comunicación Social. Y una vez, casi de casualidad, llevando a mis estudiantes a hacer una visita a la cárcel, porque donde yo vivo hay tres cárceles y es en un espacio donde advierto en un momento que había muchos egresados y estudiantes de la Universidad y en especial de nuestra facultad, trabajando como penitenciarios y algunos también como docentes. Pensé que era una interesante oportunidad para que los estudiantes de profesorado fueran a conocer algunos apectos de la educación en cárceles, porque podría ser un espacio laboral. Entiendo que es el modo de producir cambios, si quienes pasan por la universidad pueden ocupar sus puestos de trabajo sin naturalizar lo que allí se hace. Desde la cátedra y como propuesta de articulación teoría/práctica cada año visitamos escuelas y así fue como ingresamos de visita a una con sede en una unidad penitenciaria. Y una vez que entramos, lo que sucedió es que encontramos que en esa unidad penal había personas que estudiaban la carrera de Derecho como estudiantes libres de nuestra universidad y que querían que la Universidad vaya a más, "querían tener algo" con nuestra facultad. Así que a partir de ahí y casi de casualidad y sin querer y sin pensar 
quedarme, empecé a tomar en serio esto de que había que dar una respuesta y llevé la inquietud a la facultad y me pidieron que pensara la forma para dar respuesta. Y así nació el programa de educación universitaria en contextos de privación de libertad en el que ya hace 10 años que estoy trabajando. Posteriormente y a partir del trabajo en la facultad, me convocó el Rectorado para pensar una propuesta para la Universidad, desde el área de la extensión universitaria. Así que, básicamente esa es mi trayectoria en el trabajo y además soy investigadora Coordino un proyecto de investigación actualmente, enfocado en el derecho a la educación y el arte en la cárcel.

Entrevistadores - ¿Empiezan con usted, entonces, la experiencia de su Universidad con la cuestión de la educación en las cárceles?

Analía - En realidad, hubo una experiencia en mi facultad, en la década de los '90 del siglo pasado, pero se suspendió, se discontinuó porque hubo un gran motín en 1996 y a partir de ahí la facultad no fue más, aunque algunos estudiantes detenidos fueron a la sede a cursar por unos pocos años posteriormente. Por otro lado, la Facultad de Derecho, en el 2003 empieza a ofrecer carreras de grado en Azul, que es otra ciudad cercana a mi ciudad y en la que hay dos cárceles. Pero era un proyecto muy chiquitito, muy concentrado en un pequeño grupo de estudiantes. Nosotros tomamos esa experiencia como referencia, porque cuando entro a la cárcel, me encuentro con un grupo de alumnos de Derecho, como relataba. Trabajamos en esa unidad por un tiempo y lo que se hizo en estos años fue extender a seis unidades carcelarias la presencia de la Universidad. En cuatro tenemos oferta de carreras de grado. Vale la pena señalar que en la provincia de Buenos Aires hay más de 50 cárceles y en la zona que yo estoy, el servicio penitenciario bonaerense tiene nueve cárceles. Nosotros estamos llegando a seis, en cuatro se ofrecen carreras de grado, en las otras solo propuestas de extensión. No es que yo inicié, no abrí la huella, sino que ya había una. Sí abrimos otras huellas pero tomamos la experiencia de nuestra Facultad para no repetir aquellas cosas que no funcionaron y la de la Facultad de Derecho. El aula universitaria como idea y materialidad ya estaba. $Y$ entonces, bueno, la tarea fue más sostener y ampliar, incidir para que la Universidad en su conjunto advierta la relevancia del acceso a derechos educativos de las personas detenidas y se comprometa políticamente. También fue y es necesario conformar equipos de trabajo para una tarea que requiere saberes y modos específicos de afrontarla.

Entrevistadores - ¿Y cómo hoy funciona la experiencia con su Universidad en las cárceles?

Analía - Bueno, hoy la Universidad tiene dos propuestas diferentes. Una es la académica: se ofrecen carreras de grado de la Facultad de Ciencias Sociales y de la Facultad de Derecho; otra es la extensión universitaria. En la extensión universitaria hay más facultades involucradas, como la Facultad de Arte y la Facultad de Ciencias Exactas. Eventualmente puede haber alguna otra unidad académica con 
alguna actividad puntual. La Facultad de Ciencias Sociales, que es donde yo trabajo, tiene su propio programa. La Facultad de Derecho también tiene su propio programa académico de oferta de carreras de grado. Y hasta ahora lo único que había como investigación eran trabajos de tesistas de carreras de grado. Hay algunas producciones, pero no más que eso. $Y$ ahora nuestro grupo es el primero que hace investigación con temáticas de cárceles en función de la experiencia que estamos llevando adelante. Está conformado de modo interdisciplinario, por docentes de las facultades de Arte, de Derecho y de Ciencias Sociales. La particularidad de nuestro grupo no es que se decide a pensar qué pasa en la cárcel, sino que como todos estamos trabajando en el contexto de encierro, es imperativo que produzcamos conocimiento a partir de lo que estamos haciendo.

Entrevistadores - Hoy la experiencia de su Universidad hay una coordinación general ¿o no? cada uno hace sus experiencias...

Analía - Hay una coordinación por unidad académica. Sociales, Derecho. Pero yo además coordino el programa del Rectorado desde la Secretaría de Extensión. Entonces mi objetivo es coordinar con las unidades académicas, facilitando que participen a través de acompañarlos en resolver y superar múltiples obstáculos que tenemos para ingresar y permanecer en las cárceles, que entiendan la lógica con la que se habita y trabaja allí, que ofrezcan aspectos propios de las ofertas que hacen desde las unidades académicas ahora, a las personas detenidas. Obviamente, cada unidad académica tiene su propia dinámica, su autonomía y toman sus propias decisiones. Pero como yo tengo doble rol, esto de ser la responsable de mi facultad, pero también del Programa de la UNICEN, uno de los objetivos que me propuse era, no sólo ampliar el derecho del acceso a la educación, la cultura y el arte, sino también la coordinación con otras unidades académicas para potenciar la ampliación y acceso a derechos. Otro de los objetivos que propuse para el programa es establecer relaciones con otros programas universitarios, nacionales y del exterior. Digo, en ese momento yo no estaba imaginando estas conexiones, por fuera de los límites de nuestro territorio, pero sí con otros centros universitarios que, ya sea en docencia, en extensión o en investigación pudiéramos hacer en vistas a fortalecer el desarrollo del campo y de nuestro conocimiento respecto de un área en la que la UNICEN tenía muy poco desarrollo. Que es justamente el acceso a derechos... y no solo educativos, nosotros estamos pensando a la cultura en términos amplios y al arte porque, las leyes generales incorporan al arte como un derecho, sin embargo en la cárcel no existen oportunidades, entonces, uno de los planteos que hacemos es; indagar qué está pasando, qué ofertas existen, qué marco legal hay, y trabajar por el cumplimiento del acceso, de hecho al arte en la cárcel.

Entrevistadores - La experiencia Argentina con la cuestión de la Universidad en las cárceles es amplia y de larga trayectoria, que toda la parte de América del Sur comprende como una buena experiencia. ¿Por qué es muy fuerte esta idea, o esta experiencia en varias universidades, con la cuestión de implementar acciones directamente en las cárceles? 
Analía - En principio, la experiencia más resonante del país es la que desarrolla la Universidad de Buenos Aires (UBA), que a partir de 1985, a la vuelta de la democracia y en términos de ocuparse de derechos humanos la Universidad, admite a algunos estudiantes y negocia un espacio para poder tener un sitio para su propio desarrollo que es el Centro Universitario de Devoto,. Hay una linda película que se llama "No ser dios y cuidarlos"1, que es un documental sobre la historia del Centro Universitario de Devoto sin quisieran conocer este relato en primera persona. Allí se presentan entrevistas de quienes fueron los primeros presos a quienes les dan el edificio para que trabajen en su reparación y conformen un espacio para la universidad. Es muy interesante, porque ellos cuentan que cómo se suponía desde la dirección de la cárcel que no iban a hacer nada, por eso les dieron ese lugar... bueno y relatan cómo disputan el espacio y logran colocar a la Universidad dentro de la cárcel. A lo largo de los años, por ejemplo, en mi facultad, en los 90 se quiere copiar esa experiencia, pero hay un motín muy sangriento en la Unidad Penal en la que trabajaban y a la que hemos regresado hace unos pocos años. Muere gente, secuestran a una jueza, corrió sangre. Fue el motín más cruento de la historia en Argentina. En ese momento la facultad deja de ingresar a dar clases. Recién en 2008 volvemos desde mi cátedra a una visita en otra de las cárceles de la zona y posteriormente se retoma el trabajo académico.

Pero para entender cómo se amplía el trabajo de las universidades en Argentina a nivel nacional y durante el gobierno del kirschnerismo hay que advertir dos aspectos: por un lado el crecimiento de experiencias y trabajo de docentes en contextos de privación de libertad que buscaron visibilizar y reclamar por el reconocimiento de la modalidad y por otro, la oportunidad de incidir en una reforma del marco normativo que tenía como norte políticas de inclusión y que alcanzó a la educación en contextos de privación de libertad. Se incide tanto en la sanción de la Ley de Educación Nacional, en 2006 como en la reforma de la Ley de Ejecución de la pena en el año 2011. Por primera vez en una ley de educación es reconocida la modalidad de Educación en contexto de privación de libertad. Y posteriormente hay una reforma de la Ley de ejecución de la pena en la que se establece que la educación debe estar asegurada y que se tiene que facilitar su acceso, incluido el nivel superior. Asimismo se prescriben y pautan formatos que a nosotros nos sirven hoy como por ejemplo, que quienes estudian en la Universidad puedan asistir a rendir exámenes, puedan cursar y se pauta que si la persona va a la facultad, como nos sucede, quien lo acompañe no tenga que ir de uniforme, es decir, le tienen que garantizar ir sin identificar que es una persona detenida. En ese marco y en las políticas que se despliegan en los periodos de gobierno del kirschnerismo, hay un llamado a los rectores de universidades nacionales, a través del Consejo Interuniversitario Nacional - el CIN - para que se ocupen de la educación superior en la cárcel. Hay un pedido directo a que las universidades lo hagan. Por eso si uno mira, estamos hablando de 10 años y de 10 años en los que hay una cantidad importante de universidades que empezamos a tener presencia, que no es casual, no es porque justo se dio. Buena parte de las universidades han entrado a trabajar en las cárceles desde la extensión, y

\footnotetext{
1 "No ser Dios y cuidarlos. Una película de Juan Carlos Andrade y Dieguillo Fernández. Película completa em https://www.youtube.com/watch?v=Y-nxcEZXo9A
} 
muchas han logrado luego instalar una oferta de grado. Nosotros entramos por la oferta de carreras de grado.

Me parece que vale la pena revisar algunos aspectos respecto de la universidad argentina. Uno de los más relevantes a destacar es que a pesar de los múltiples ataques que viene sufriendo en el periodo de gobierno del presidente Macri, aún sigue siendo pública y gratuita y de ingreso irrestricto. No obstante sigue teniendo una matriz elitista que la hace selectiva y excluyente si se mira quiénes pueden llegar y la permanencia y promoción del estudiantado. Si pensamos en la Universidad en la cárcel, tiene que pensarse además que la tarea incluye alcanzar a la población que no puede o no elige ser estudiante pero puede ser destinataria de propuestas que democraticen el acceso a los bienes materiales y simbólicos que se producen y se disponen. Entonces, ¿cómo llego a ellos? a través de la extensión: una oferta cultural, una oferta artística, charlas académicas, jornadas, festivales. Entonces las universidades nacionales, diría yo, entre el 2008, 2010 hasta el final del gobierno de Cristina Fernández de Kirchner en 2015, que fueron alentadas a través de una política que buscaba la inclusión, a ser partícipes y responsables de llegar a muchos territorios, entre ellos, las cárceles. También es cierto que no se incrementó al presupuesto, o sea, que luego que las universidades empiezan a trabajar porque hay que hacerlo, lo que tuvimos que hacer es pelear adentro de la Universidad por el dinero, ¿sí? no hubo un presupuesto de los ministerios... Sí a través de proyectos que se concursan a través de la secretaria de políticas universitarias, que se convocan y que son a término de un año. Cuando se terminan es complejo garantizar la continuidad. Creo que ahí lo bueno de esas políticas del Ministerio fue que impulsó a trabajar, lo malo fue que nos dejó trabajando sin nada. Además, teniendo en cuenta la historia de la universidad, el estudiantado compuesto por personas detenidas es algo que desancla tradiciones y visiones. Hay que conquistar voluntades y recursos. Pero bueno, creo que por eso también muchos docentes universitarios estamos trabajando en la cárcel. Porque hay una voluntad política de llegar y permanecer en estos espacios.

Entrevistadores - En el caso de Brasil, tenemos casi el $70 \%$ de las personas que están en las cárceles que no han concluido la enseñanza fundamental [primaria], por ejemplo. Para nosotros la prioridad es la educación básica. Yo estoy pensando, por ejemplo, ¿cómo es la educación básica y cuál es ese contexto, en Argentina? Porque, pensá, por ejemplo, que para nosotros en la política nacional no es tan prioridad pensar en una formación [un grado], una enseñanza dentro de las cárceles porque la cuestión básica que tenemos que hacerla desde la alfabetización de las personas a la conclusión de la educación básica.

Analía - Bueno, en realidad esto fue en parte dar respuesta a demandas de personas detenidas que le piden a las universidades poder matricularse. Además, del consejo de rectores se plantea una demanda específica de mayor compromiso con el territorio. No quiere decir que adentro de la cárcel no haya otras problemáticas, como por ejemplo, que cada vez son más jóvenes los que ingresan y que hay muchas personas que nunca completaron la escolaridad, o que no saben leer, ni escribir. Siempre 
la Universidad, digamos, en términos de pirámide de población, recibe mucho menos personas que las que tendrían edad para estar en la Universidad. La educación, en general, se ha... entiendo yo que ha habido cambios de políticas educativas que no fueron superadoras en la década de los '90, hubo una ley que se derogó con la sanción de la del año 2006, que fue muy dañina para la estructura del sistema.

A partir de la sanción de la Ley Nacional que está vigente, hubo políticas de inclusión, hubo mayor apertura hacia financiamiento, hacia materiales didácticos. Se cambió la estructura en el sistema educativo en seis años de primaria, seis años de secundaria. La secundaria se hizo obligatoria. Se sigue manteniendo la educación superior pública y gratuita. La Universidad sigue siendo uno de los baluartes de la Argentina, como educación gratuita, no sólo pública, pero hay muchas deudas del sistema educativo en términos de resultados. Durante el periodo de gobierno de Kirchner y de Fernández, hubo mucha mejora, pero desde que llegó Macri al gobierno, el gobierno actual, se marca una política hacia la derecha sin matices. Este período puede caracterizarse por estar llevando adelante políticas económicas que traen endeudamiento del país con organismos internacionales, transferencia de recursos a privados; quita de subsidios, cierre de industrias, crecimiento de la desocupación y el desempleo, falta de oportunidades, crecimiento de la pobreza. En relación a la educación, y en particular la universidad, entre las primeras consignas del presidente, aún en campaña, fue decir "¿qué es esto de universidades por todos lados?" En los años de su gobierno se ha deteriorado profundamente el presupuesto universitario y el crecimiento en número de universidades con una perspectiva inclusiva y destinada a sectores históricamente excluidos está siendo puesta en jaque. Además se advierte un desprecio profundo por la tarea de los docentes, por los resultados, por los formatos y un vaciamiento de todos los programas que se habían creado para sostener políticas inclusivas como la educación sexual integral; políticas que tenían que ver con la discapacidad, con la formación permanente de los docentes de modo gratuito, porque había plataformas, cursos, especializaciones regulares en términos de continuidad y de largo plazo que ofrecía el Estado. Sin embargo, todo eso fue rápidamente vaciado en términos de desfinanciamiento. Entonces, las políticas de distribución de libros en las bibliotecas, la políticas inclusivas de acceso a tecnologías y conectividad, de acceso a la salud, al deporte dejan de estar. Uno de los programas que creó el Kirschnerismo fue el "Conectar igualdad" donde se entregaban computadoras a todos los y las estudiantes, no importaba al sector social de procedencia, solo que asistiera a una escuela secundaria pública y a todos los estudiantes de los profesorados públicos y a sus docentes. Entonces había una entrega de equipos para estar conectados a intranet pero también con acceso a internet en todas las escuelas. Bueno, este fue uno de los primeros programas que dejó de funcionar, siempre con argumentos de malos resultados y se empezó a reemplazar por programas que son ofrecidos por empresas y ahora pagados por el Estado. Digamos, grandes negocios respecto de la escolaridad, una muestra clara del modo en que se ve a la educación como mercancía y no como derecho social. Lo que queda claro es que también, durante el gobierno kirschnerista, grandes sectores de la población no lograron superar su condición de pobreza estructural y siguieron siendo aquellos que son prisionizados. Digamos que políticas de seguridad que no se resolvieron en ese período tampoco, donde en los 
barrios más pobres, sobre todo de lo que se llama conurbano, que es lo que rodea la capital del país, que es enorme, súper poblada y que tiene terribles problemas de infraestructura, en términos de luz, agua, cloacas y de trabajo, esos sectores siguieron siendo vulnerados permanentemente y entre ellos el acceso a la educación y al trabajo. De allí recibimos mayoritariamente nuestros estudiantes, las personas que viven en las cárceles de la ciudad donde yo estoy vienen del conurbano y son jóvenes y muchos de ellos son escolarizado adentro de la cárcel y han estado viviendo en la calle desde que tienen ocho, nueve y diez años. $Y$ fueron, y si tienen hoy veinte o veinticinco son de este período, no estamos hablando solo del menemismo². Es decir que las políticas públicas fueron muy importantes, pero no suficientes.

Entrevistadores - Hay varias universidades que tienen experiencia con la educación en las cárceles. ¿Hay alguna articulación de estas universidades sobre la cuestión del tema, o no?

Analía - Bien, sí, nosotros participamos allí por 2010 en una incipiente mesa interuniversitaria nacional de universidades que trabajamos en contexto de privación de libertad. Esta fue una convocatoria que hizo la Universidad de Cuyo que está en la ciudad de Mendoza, avalada por el Ministerio de Educación porque la provincia en ese momento tenía un terrible problema de denuncias e intervino la Corte Interamericana de derechos humanos Como una de las acciones, la Gobernación y el Poder Judicial le piden a la Universidad que intervenga y comienzan su trabajo en la cárcel. Son ellos y en ese contexto, acompañados por el Ministerio de Educación de la Nación que convocan a ese primer encuentro en el que éramos muy poquitas las universidades presentes $A$ partir de ahí, en diferentes momentos nos hemos reunido. Lo que tenemos es, primero, un reconocimiento de quienes somos bastante bien, qué hacemos en las cárceles. Tenemos un estado de situación de lo que está pasando en el país. La última mesa que fue la quinta, tuvo lugar en la ciudad de Rosario en 2018. Allí quedamos en un acuerdo para conformar encuentros regionales, y ya se establecieron lazos y estamos trabajando en eso para compartir intereses, preocupaciones y también para compartir información. Para respaldarnos frente a situaciones que no sabemos cómo manejar, porque en realidad hay muchas cosas que desconocemos o que son novedosas y que no han tenido una respuesta. Entonces la experiencia de los compañeros de otras universidades compartir cómo proceden o qué protocolo han desarrollado y les da resultado, permite avanzar sin pasar por obstáculos que pueden ser evitados, son herramientas que podemos compartir y también la circulación de producción científica. Es decir, compartir artículos, invitarnos a publicar y producir cosas compartidas, eso está empezando a funcionar un poco mejor. Yo diría en los últimos tres, cuatro años-funciona mejor la red ${ }^{3}$.

Somos conscientes que lo que sucede en términos de avance del punitivismo y crecimiento del encarcelamiento no sucede solamente en Argentina, que es un problema mundial y es parte del avance

\footnotetext{
${ }^{2}$ Gobierno del presidente Carlos Menem 1989 - 1999.

3 Puede contactar a la Red Educación en Contextos de Encierro de Argentina a traves del correo electrónico: rededucacionyencierro@gmail.com
} 
del neoliberalismo que encuentra a la cárcel como parte de su modo de gobierno. Necesitamos consolidar proyectos que nos fortalezcan para poder trabajar colectivamente en la transformación de estas realidades que nos pretenden doblegar.

Entrevistadores - Otra experiencia que en varios países de América del Sur se habla sobre las bibliotecas en las cárceles de Argentina. ¿Conoces experiencias, qué sabes de eso?

Analía - Sí, conozco... escuché a una bibliotecaria del sur de nuestro país presentar la experiencia de un encuentro que hicimos nosotros mismos en 2012 en Olavarría, presentó sobre los equipos que llegaron a las cárceles federales con un proyecto del Ministerio de la Educación, las "Bibliotecas abiertas" junto con la creación de un puesto de bibliotecario/a; de cómo se distribuían y la promoción de la lectura al interior de las unidades penitenciarias, pero no tengo experiencia de haber estado cerca, y es más, he promovido dentro las cárceles bibliotecas aunque no han tenido gran impacto. De ese proyecto que mencionás, solo esa persona que escuché contar que ella era la bibliotecaria y lo lleva adelante, lo sigue sosteniendo, pero nada más porque nosotros no hemos tenido contacto con el Ministerio de Educación de la Nación. El Ministerio de Educación de la Nación en el área del contexto de encierro está destinado a niveles primario y secundario y la Universidad por la misma estructura orgánico-administrativa está separada, depende orgánicamente de la Secretaría de Políticas Universitarias.

Entrevistadores -Para concluir aquella cuestión ante la coyuntura política de Argentina de acirramento del neoliberalismo y sus consecuencias sociales...

del acirramiento del neoliberalismo y de la represión, sobre los pobres y sus consecuencias políticas económicas culturales, ¿cuáles son tus perspectivas de los desafíos de la educación?

Analía - La primera situación que se presenta con esta avanzada de la nueva derecha es, la represión y la prisionización. Es decir que personas que podrían permanecer fuera de la cárcel hasta tanto sea su juicio, están detenidas. Esto ha generado una sobrepoblación y una condición de vida dentro de la cárcel muy difícil. Muy difícil en general, por la falta de alimentos, por la falta de salud, por la infraestructura de las cárceles que no están preparadas para duplicar la cantidad de gente que tienen respecto de la capacidad real. Por otro lado, las plazas educativas y los espacios físicos siguen siendo los mismos. Entonces el primer desafío, me parece, es que los educadores deberíamos visibilizar esto, porque hasta ahora me parece que la actitud ha sido, "bueno, nosotros podemos tomar hasta tantos" y "bueno, tomamos lista de espera"... Me parece que hay que empezar a comprometerse en acompañar los reclamos, en poder mostrar lo que sucede en el interior, tras los muros. El riesgo siempre está en que no los dejen ingresar más, o que consigan las herramientas para que las instituciones se sometan a la voluntad del servicio penitenciario, colocar mayor control, mayores obstáculos. Me parece que otro 
de los desafíos de la educación, además está en formar una mirada más crítica sobre la realidad, más interesada en la transformación que en el contenido en sí mismo, porque no digo que los docentes no enseñen, sino que enseñen con sentido de transformación, con sentido de conquistar otro espacio para sí y para el resto. Me parece que tenemos que fortalecer eso incluso en los cuerpos docentes. Me parece que la deuda está también en la formación, en los modos que formamos a quienes ingresan a trabajar, porque si los docentes no tienen claridad sobre qué pueden hacer, es decir, qué se puede decir qué no, que se puede poner el límite, que se puede reclamar un trato digno, no estarían pensando a la tarea como parte de la formación ciudadana. ... Entender que la tarea de la educación no está al servicio de la rehabilitación, que la educación está para desafiar la imposición, la dominación, me parece que es una cuestión de perspectiva ideológico- política. Por el otro camino, la educación asume otros sentidos, se termina en una cuestión técnico instrumental de alfabetización para la dominación, finalmente para la sumisión. Entonces creo que el desafío es trabajar y de modo muy inteligente además, para que podamos permanecer. Claro, hay que construir la táctica dentro de la cárcel para permanecer más allá de la adversidad, pero no negociar entregando, justamente, este núcleo duro que es el para qué uno está ahí adentro, me parece que lo que hay que proteger con todas nuestras fuerzas desde la claridad conceptual y nuestras prácticas concretas que no pueden ir en contrario. Podemos negociar, pero eso no se negocia, digamos, habrá que estar muy alertas. Creo que el miedo es una de las estrategias más fuertes que está avanzando en la sociedad, en la instalación del miedo, el miedo a perder el trabajo, el miedo a no poder, que digamos "qué voy a hacer yo si esto siempre fue así...", entonces, fortalecernos también como colectivo. No se puede pensar esto como una cuestión individual en ningún sentido. $Y$ ahora mucho menos, por eso es importante comprometer la voluntad política de las universidades para sostenernos, frente a cualquier eventualidad que pueda suceder con nuestro trabajo, a sostenernos en términos de nuestros reclamos como justos y en nombre de los derechos humanos.

Entrevistadores - Sí, tener respaldo.

Analía - En ese sentido que nos sostengan, y poder seguir construyendo alternativas, mostrar trabajo serio y conseguir respaldo político, para poder no hacer la vista a un lado con situaciones que tenemos que testimoniar, porque si no ¿quién lo va a hacer?

Entrevistadores - Muchas gracias. 


\section{Como citar este documento:}

GODINHO, Ana Claudia Ferreira; JULIÃO, Elionaldo Fernandes; UMPIERREZ, Analía. A experiencia Argentina de oferta de Ensino Superior no Sistema Prisional - entrevista com Analía Umpierrez. Reflexão e Ação, Santa Cruz do Sul, v. 27, n. 2, abr. 2019. ISSN 1982-9949. Disponível em: <https://online.unisc.br/seer/index.php/reflex/article/view/13144>. Acesso em: doi:https://doi.org/10.17058/rea.v27i2.13144. 